\title{
AVALIAÇÃO QUANTIQUALITATIVA E FITOSSANITÁRIA DAS ESPÉCIES VEGETAIS DO CAMPUS IV - UEPB: UM ENFOQUE SUSTENTÁVEL
}

\section{AVALIAÇAO QUANTIQUALITATIVA E FITOSSANITÁRIA DAS ESPECIES VEGETALES DO CAMPUS IV - UEPB: UM ENFOQUE SUSTENTAVEL}

\section{EVALUATION QUANTIQUALITATIVE AND PHYTOSANITARY OF VEGETABILES SPECIES CAMPUS IV - UEPB: A SUSTENAIBLE APPROACH}

\author{
Maeli Freitas SOUSA ${ }^{1}$ \\ Mirtes Raísla Fernandes DUTRA ${ }^{2}$ \\ Francineide Pereira SILVA ${ }^{3}$ \\ Thiago Pereira SOUSA ${ }^{4}$ \\ Fabiana Xavier COSTA ${ }^{5}$
}

RESUMO: Objetivou-se com este trabalho avaliar quantiqualitativamente e fitossanitariamente as espécies vegetais do Campus IV da UEPB em Catolé do Rocha - PB, onde todo resultado foi transmitido para comunidade acadêmica, através de um processo de sensibilização envolvendo a educação ambiental e no segundo momento deste trabalho implantar uma rearborização no referido Campus. A pesquisa foi realizada no período de agosto/2010 a fevereiro/2013, desenvolvida por setores, sendo eles: bovinocultura, olericultura, caprinocultura, riacho agom e projeto de palmas do Campus IV da Universidade Estadual da Paraíba. Nos cinco setores estudados, foram catalogadas 13.677 plantas de 46 espécies diferentes. As seis espécies mais freqüentes perfizeram $75 \%$ do total de plantas. Sendo o marmeleiro (Cydonia oblonga) a espécie de maior frequiência, com 4.358 plantas, representando 31,86\% do total, seguindo a jurema preta (Cydonia oblonga) com 1.648 plantas, representando 12\%, a jurema (Mimosa hostilis) com 1.596 plantas representando, 11,6\% o mororó (Bauhinia forficata) com 941 plantas representando 6,8\%, a orelha de macaco (Enterolobium contortisiliquum) com 897 plantas representando 6,5\%, o mufumbo (Combretum leprosum) com 824 plantas representando 6,0\%.Há alta diversidade de espécies nas áreas arborizadas. Com a alta diversidade de espécies, apesar de grandes problemas de vandalismo e fitossanidade encontrados nos setores avaliados, indica qualidade na estutura física e ambiental. O conhecimento da diversidade florística avaliado é de fundamental importância para a estrutura vegetativa. $\mathrm{O}$ marmeleiro foi a espécie mais encontrada no Campus IV.

\footnotetext{
${ }^{1}$ Departamento de Agrárias e Exatas. Licenciatura em Ciências Agrárias. Universidade Estadual da Paraíba (UEPB). maeli_freitas22@hotmail.com

${ }^{2}$ Departamento de Agrárias e Exatas. Licenciatura em Ciências Agrárias. Universidade Estadual da Paraíba (UEPB). mirtes_raislla@hotmail.com

${ }^{3}$ Departamento de Agrárias e Exatas. Licenciatura em Ciências Agrárias. Universidade Estadual da Paraíba (UEPB). rochafrance@ hotmail.com

${ }^{4}$ Departamento de Agrárias e Exatas. Licenciatura em Ciências Agrárias. Universidade Estadual da Paraíba (UEPB). tiagojd2009@hotmail.com

${ }^{5}$ Departamento de Agrárias e Exatas. Licenciatura em Ciências Agrárias. Universidade Estadual da Paraíba (UEPB). fabyxavierster@gmail.com
} 
Palavras-chave: Educação ambiental, composição florística, arborização.

RESUMEN: El objetivo de este estudio fue evaluar cuanti fitossanitariamente y especies de plantas Campus IV UEPB Catolé do Rocha - PB, donde se transmitieron todos los resultados a la comunidad académica a través de un proceso de sensibilización relacionadas con la educación ambiental y la segunda vez que este trabajo implementar una reforestación en ese campus. La encuesta se realizó entre Agosto/2010 a fevereiro/2013 desarrollado por sectores, a saber: el ganado, la horticultura, la cabra, Agom arroyo y las palmas de proyectos IV Campus de la Universidad del Estado de Paraíba . En los cinco sectores estudiados, se catalogaron 13.677 plantas de 46 especies diferentes. Las seis especies más abundantes representaron el $75 \%$ del total de plantas. Siendo la membrillo (Cydonia oblonga) las especies con la frecuencia más alta, con 4.358 plantas, lo que representa $31,86 \%$ del total, el jurema negro (Cydonia oblonga) con 1.648 plantas, que representan el $12 \%$ jurema (Mimosa hostilis) con 1.596 plantas que representa el 11,6\% del Mororó (forficata Bauhinia) con 941 plantass que representan el 6,8\%, el mono oído (nterolobium contortisiliquum) con 897 plantas que representan el 6,5\%, el mufumbo (Combretum leproso) con 824 plantas que representan el $60 \%$. hace una alta diversidad de especies en las zonas boscosas. Con gran diversidad de especies a pesar de los grandes problemas de vandalismo y plantas que se encuentran en los sectores evaluados, indica el estutura calidad física y ambiental. Conocimiento de la diversidad florística de tasación es de fundamental importancia para la estructura vegetativa. El membrillo es la especie más común en el Campus IV .

Palabras clave: Educación Ambiental, composición florística, la forestación .

ABSTRACT: The objective of this study was to evaluate qualitativequanti and plant species Campus IV UEPB Catolé do Rocha - PB , where all results were transmitted to the academic community through a sensitization process involving environmental education and the second time this work implement a reforestation in that campus. The survey was conducted from August/2010 to February/2013 developed by sectors, namely: cattle, horticulture, goat, agom creek and project palms IV Campus of the State University of Paraiba. In the five sectors studied, 13,677 plants of 46 different species have been cataloged. The six most abundant species accounted for $75 \%$ of total plants. Being the quince (Cydonia oblonga) the species with the highest frequency, with 4,358 plants , representing $31.86 \%$ of the total, the black jurema ( Cydonia oblonga) with 1,648 plants, representing $12 \%$ jurema ( Mimosa hostilis ) with 1,596 plants representing $11.6 \%$ the mororó (forficata Bauhinia) with 941 plants representing $6.8 \%$, the ear monkey (Enterolobium contortisiliquum) with 897 plants representing $6.5 \%$, the mufumbo (Combretum leprosum) with 824 plants representing $60 \%$ . Ago high diversity of species in the wooded areas. With high species diversity despite major problems of vandalism and plant found in the sectors evaluated, indicates the physical and environmental quality structure. Knowledge of floristic diversity assessed is of fundamental importance for the vegetative structure. The quince was the species most commonly found in the Campus IV.

Keywords: Environmental Education, floristic composition, afforestation. 


\section{1- INTRODUÇÃO}

O meio ambiente comumente chamado apenas de ambiente, envolve todas as coisas vivas (biótica) e não vivas (abiótica) que ocorrem na terra ou algumas regiões dela, que afetam os ecossistemas e a vida dos humanos. É o conjunto de condições, leis, influencias e infraestrutura de ordem física, química e biológica, que permite abrigar e reger a vida em todas as suas formas.

Infelizmente o nosso planeta é afetado por vários problemas ambientais, muitos deles provocados por diversas ações humanas. Estes problemas afetam a fauna, flora, solo, água, ar e outros. O desmatamento e um dos fatores que causam impacto ambiental.

Segundo Science, (2012), varias espécies vegetais já foram extintas por causa do desmatamento.

O Reino Plantal, (vegetal) é um dos maiores grupos de seres vivos na Terra com cerca de 400.000 espécies conhecidas, incluindo uma grande variedade de ervas, árvores, arbustos, plantas microscópicas, etc. São, em geral, organismos autotróficos cujas células incluem um ou mais organelas especializados na produção de material orgânico a partir de material inorgânico e da energia solar, os cloroplastos. Cerca de 300 espécies conhecida de plantas não realiza a fotossíntese, sendo, pelo contrário parasitas de plantas fotossintéticas.

A educação ambiental é o único meio, para que possamos nos conscientizarmos. A sua principal função é promover á preservação do meio ambiente e a sua utilização sustentável.

Entende-se que educação ambiental pode ser aplicada de diversas formas, mais com uma única finalidade construir valores sociais, conhecimento, habilidades e competências voltados para a conservação do meio ambiente et al DIAS, (2004).

Segundo ASSIS (2000), sustentabilidade e um termo usado os para definir ações e atividades humanas que visam suprir as necessidades atuais dos seres humanos, sem comprometer o futuro das próximas gerações, ou seja, sustentabilidade esta diretamente relacionada ao desenvolvimento econômico e material sem agredir o meio ambiente.

Diante da situação em que se encontra as espécies arbóreas do Campus -IV, onde alunos usam as mesmas como varal para roupas, armadores de redes,e outros tipos de 
finalidade alem de estarem plantadas em locais não adequados, próximo a rede elétrica e hidráulica e a estradas de acesso ao Campus, visto tudo isso foi realizado esse trabalho no intuito de despertar a comunidade acadêmica a respeito da real situação do Campus.

Diante do exposto objetivou-se com esse trabalho identificar de forma quantiqualitativa e fitossanitárias as espécies vegetais existentes nos setores (Bovinocultura, Olericultura (mandala), Caprino cultura, Riacho Agom e Projeto de Palmas (área de canhão I e II e área do milharal) do Campus IV da Universidade Estadual da Paraíba, no município de Catolé do Rocha-PB.

\section{METODOLOGIA}

\subsection{Local}

O presente trabalho foi realizado no Campus IV da Universidade Estadual da Paraíba no município de Catolé do Rocha - PB, com 104 hectares aproximadamente, situado a $272 \mathrm{~m}$ de altitude, 6 $6^{\circ} 20^{\prime} 38^{\prime \prime} \mathrm{S}$ Latitude e $37^{\circ} 44^{\prime} 48^{\prime \prime}$ O Longitude (Figura 1), no período compreendido entre agosto de 2010 a fevereiro de 2013.

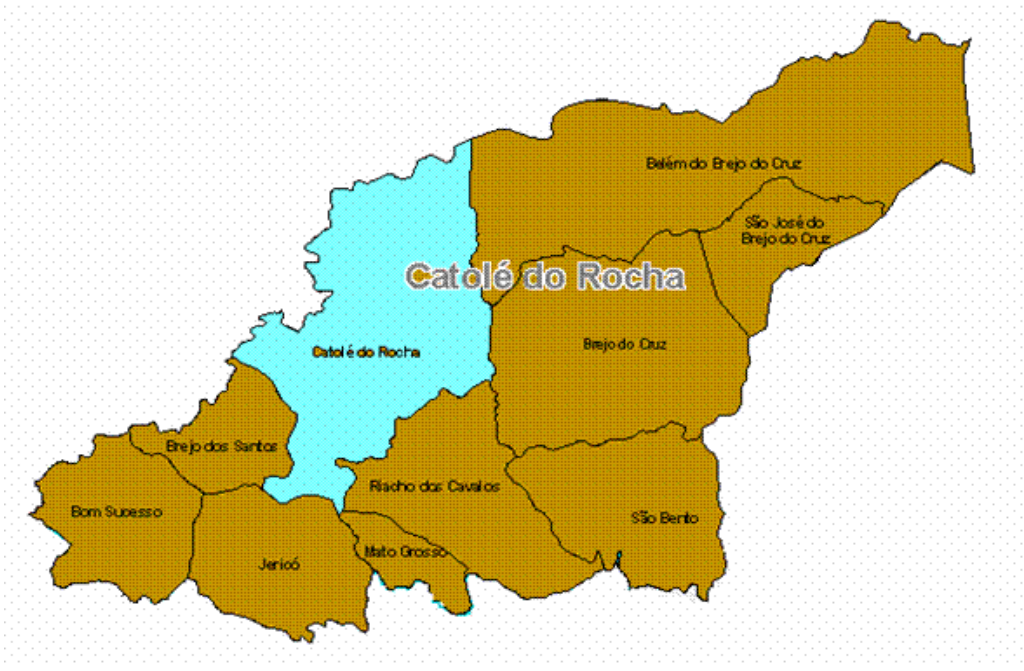

Figura 1- Mapa da micro região de Catolé do Rocha/ PB.

A pesquisa foi realizada por setores e áreas sendo eles: Bovinocultura, Olericultura (Mandala) Caprinocultura, Riacho Agom e Projeto de Palmas (área de canhão I e II e área do milharal). 


\subsection{Momentos da pesquisa}

A pesquisa foi dividida em três momentos, sendo eles:

\subsubsection{Primeiro momento}

No primeiro momento foi feito um levantamento quantiqualitativo e fitossanitário das espécies vegetais.

A levantamento quantitativo refere-se a quantidade de espécies vegetais existentes em cada área trabalhado no Campus. O qualitativo refere-se à conservação das espécies, ou seja, se há algum problema de vandalismo, implantação inadequada, desidratação, falta de poda, entre outros (Figura 2). O levantamento fitossanitário refere-se à incidência de doenças das espécies, no entanto será observado as espécies atacadas por fungos, bactérias ,ataque cupins, bem como outros parasitas e, assim, será diagnosticado o tipo de parasita e doença causada pelos o mesmos.

Foi feito nesse primeiro momento a devida avaliação das espécies atacadas por pragas e as que não forem recuperadas serão substituídas num outro momento da pesquisa.

Todos os momentos da pesquisa foram registrados via câmera fotográfica e foi utilizado um mapa do Campus para melhor localização dos setores trabalhados.

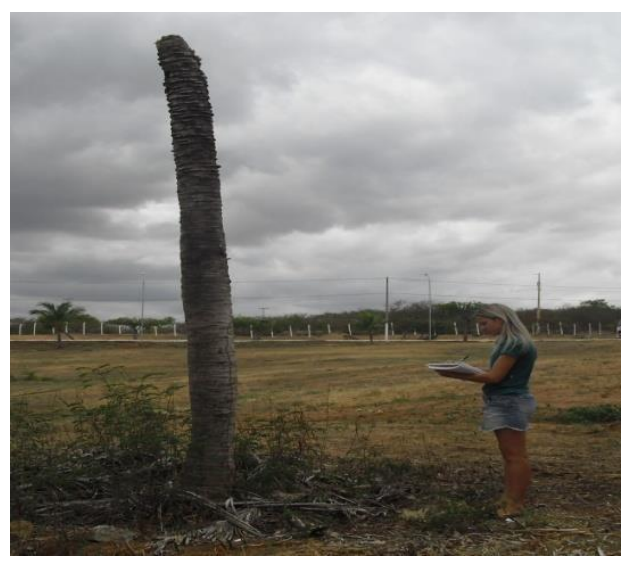

Figura 2- Levantamento quantiqualitativo das espécies vegetais, UEPB, Catolé do Rocha/ PB, 2010 / 2013. 


\subsubsection{Segundo momento}

No segundo momento foi feito um trabalho de educação ambiental, envolvendo toda a comunidade do Campus, como alunos (Figura 3), professores (Figura 4) e funcionários (Figura 5), para que se possam apresentar os resultados das espécies vegetais encontrados no Campus e, assim, procurar sensibilizá-los a respeito da real situação delas, com o objetivo de conseguir colaboração na preservação das mesmas, bem como mantê-los informados dos possíveis problemas encontrados.

Para isso, foram utilizados cartazes, fotos, álbum seriado, palestras e panfletos.

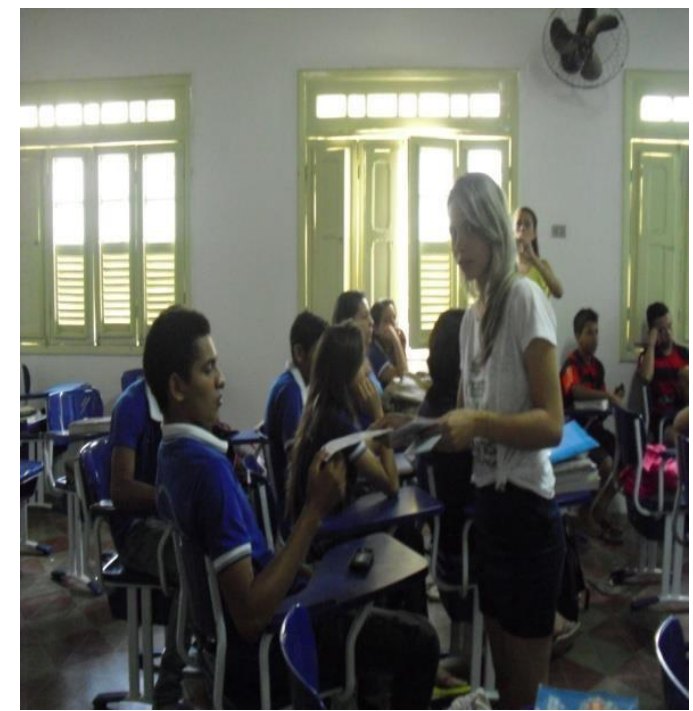

Figura 3- Educação ambiental com a comunidade acadêmica (alunos). UEPB, Catolé do Rocha - PB, 2010 / 2013.

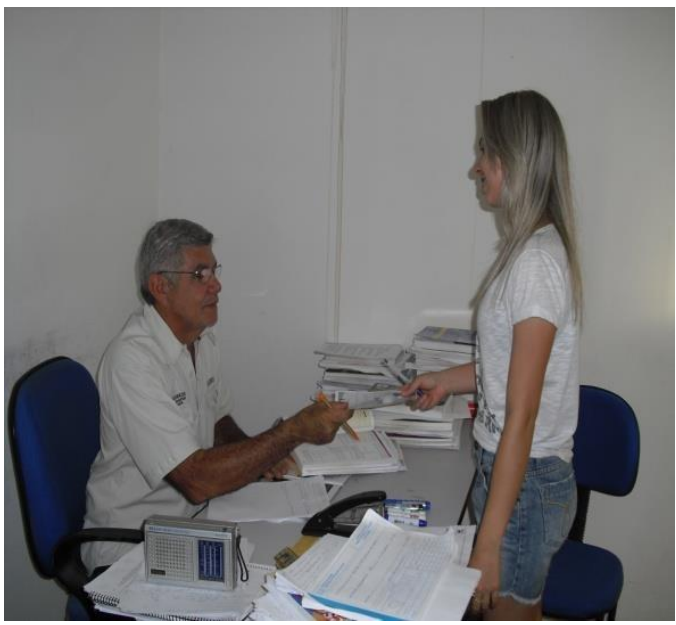


Figura 4 - Educação ambiental com a comunidade acadêmica (professores). UEPB, Catolé do Rocha-PB, 2010 / 2013.

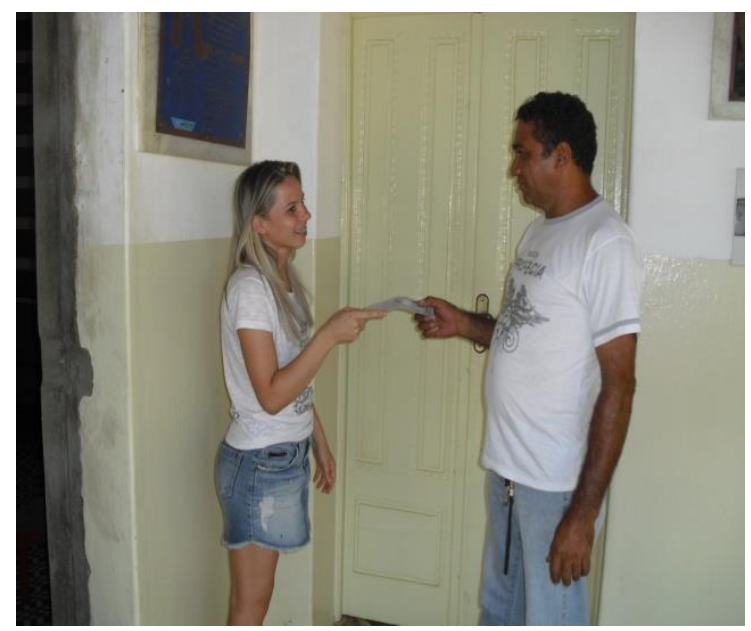

Figura 5 - Educação ambiental com a comunidade acadêmica (funcionários). UEPB, Catolé do Rocha - PB, 2010 / 2013.

\subsubsection{Terceiro momento}

O terceiro momento não pôde ser realizado em seguida o levantamento, por causa da falta de chuvas, mas num futuro próximo, será feito a provável substituição de espécies vegetais doentes que não puderam ser recuperadas, mortas, bem como outros problemas. Será feito a arborização de áreas desérticas, com o objetivo de deixar todo o Campus arborizado quantitativamente, qualitativamente e fitosanitariamente, mantendo, assim a preservação de todas as espécies existentes.

Para isso serão adquiridas mudas referentes às espécies que serão substituídas e plantadas.

\section{RESULTADOS E DISCUSSÃO}

Nos cinco setores estudados, foram apresentadas 13.677 plantas, de 46 espécies diferentes. As seis espécies mais freqüentes perfizeram $75 \%$ do total de plantas. Sendo o marmeleiro (Cydonia oblonga) a espécie de maior freqüência, com 4.358 indivíduos representando, $31,86 \%$ do total seguindo a jurema preta (Cydonia oblonga) com 1.648 de 
indivíduos, representando 12\%\%, a jurema (Mimosa hostilis) com 1.596 indivíduos, representando, 11,6\% o mororó (Bauhiniaforficata) com 941 indivíduos, representando 6,8\% a orelha de macaco (Enterolobium contortisiliquum)com 897 indivíduos , representando 6,5\% o mufumbo (Combretum leprosum) com 824 indivíduos, representando 6,0\%. Foram observadas 35 espécies com apenas um indivíduo correspondendo a $0,259 \%$ da população amostrada.

\section{1-Setor de Bovinocultura}

Ao longo do desenvolvimento desse trabalho foram encontradas grandes variedades de espécies nativas, de acordo com a (tabela 1) no setor de bovinocultura foram catalogadas um total de 5.333 plantas, representando $38,99 \%$ do total encontrado onde o marmeleiro ( Cydonia oblonga ) foi a espécie de maior freqüência ,( com 2.201 plantas), seguido da jurema preta (Mimosa tenuiflora Cydonia oblonga) (com 1.028 plantas), a orelha de macaco (Prosopis ruscifalia) com (896 plantas), a algaroba ( Prosopisruscifalia) com ( 464 plantas), o pinhão branco (Jatropha molissima) (com 252 plantas).

De acordo com o levantamento qualitativo, conforme a (tabela 1), os principais vandalismos encontrados foram: cortes agressivos e extravagantes, grande quantidade de lixo e plásticos entrelaçados nas plantas. Os problemas de fitossanidade e nutricional mais freqüentem foram: periderme desidratada e ataque de cupins.

De acordo com Gurgel et. al ( 2009),espécies exóticas como o Azadirachta indica A .Juss (Nim indiano) e o Fícus benjamina Linn (Fícus) são algumas das espécies mais abundantes utilizadas atualmente no trabalho de arborização na cidade de Cajazeiras.

Dorneles et .al (2013), recomendaram o número de 10 a 20 espécies para compor a arborização de uma cidade, entretanto, a baixa freqüência de algumas espécies torna-se fator preocupante, uma vez que é interessante e conveniente que se mantenha um número de espécies representativas de biodiversidade como forma de visar as faces estéticas e paisagísticas do local.

Oliveira et al (2011), relata que outro aspecto importante a ser considerado é que estão diretamente relacionadas à conservação da diversidade da fauna local, servindo de refúgio para as espécies remanescentes das cidades . 
Tabela1. Levantamento quantiqualitativo e fitossanitário das espécies vegetais encontrados no setor de bovinocultura do campus IV da UEPB, Catolé do Rocha-PB, 2010/2013.

\begin{tabular}{|c|c|c|c|c|}
\hline Nome Popular & Nome Cientifico & Vandalismo & $\begin{array}{c}\text { Fitossanidade e } \\
\text { nutrição }\end{array}$ & $\begin{array}{l}N^{o} \text { de } \\
\text { Plantas }\end{array}$ \\
\hline Acerola & Mapighia glabalinn & $\begin{array}{l}\text { Cortes, } \\
\text { pendurado }\end{array}$ & Periderme desidratada & 04 \\
\hline Agodão Bravo & Ipomoea cárnea & Lixo ao redor & Desidratação & 01 \\
\hline Angico & Anadenanthera falcata & Não identificado & Ataque de cupins & 03 \\
\hline Algaroba & Prosopis ruscifalia & Cortes agressivo,lixo & Periderme desidratada & 464 \\
\hline Aroeira Branca & Lithraea molleodes & Não identificado & $\begin{array}{l}\text { Fungos, periderme } \\
\text { desidratada, }\end{array}$ & 04 \\
\hline Aroeira Roxa & Schinus terebinthfolia & Não identificado & Periderme desidratada & 03 \\
\hline Cajueiro & Onorcadiumo ccidetale & Cortes extravagantes & Periderme desidratada & 01 \\
\hline$\overline{\text { Cajarana }}$ & $\overline{\text { Spondias lútea }}$ & Cortes extravagantes & Não identificado & 01 \\
\hline Coqueiro & Cocos nucifera & Poda inadequada & Periderme desidratada & 01 \\
\hline Catingueira & $\begin{array}{l}\text { Caesalpinia } \\
\text { pyramidalistui }\end{array}$ & $\begin{array}{l}\text { Polda inadequada e cortes } \\
\text { agressivos }\end{array}$ & Ataque de cupim & 09 \\
\hline Café do mato & Cordiae calyculata & Não identificado & Não identificado & 52 \\
\hline Carnaúba & Corpernicia prunifera & Não identificado & Ataque de cupins & 03 \\
\hline Cumarú & Dipteryx odorata & Não identificado & Ataque de cupins & 01 \\
\hline Favela & $\begin{array}{l}\text { Cnidoscolu } \\
\text { sphyllacanthus }\end{array}$ & Não identificado & $\begin{array}{l}\text { Ataque de formigas e } \\
\text { cupins }\end{array}$ & 06 \\
\hline Ferruginha & NÃO ENCONTRADO & , Cortes extravagantes & Não identificado & 03 \\
\hline Graviola & Annona muricata & Cortes extravagantes & Periderme desidratada & 01 \\
\hline Goiabeira & Psidium guajava & Cortes agressivos & Periderme desidratada & 07 \\
\hline Limão & Citrus Limonium & Cortes & Não identificado & 01 \\
\hline Linhaça & Linum usitatissimum L. & Arames e lixo & Não identificado & 01 \\
\hline Juazeiro & Ziziphusjo azeiro Mart. & Cotes agressivos & Ataque de cupins & 32 \\
\hline Jucá & Libidiia Ferrea & Arames,cortesagressivos & Desidratação & 54 \\
\hline Jurema Branca & Piptadeni astipulacea & Não identificado & Periderme desidratada & 13 \\
\hline Jurema Preta & $\begin{array}{l}\text { Mimosa tenuiflora } \\
\text { Cydonia oblonga }\end{array}$ & $\begin{array}{l}\text { Cortes agressivos, lixo } \\
\text { veterinário }\end{array}$ & $\begin{array}{l}\text { Desidratação, fungos } \\
\text { (miconisa) }\end{array}$ & 1028 \\
\hline Fava de Boi & Canavalia Maritima & Cortes & $\begin{array}{l}\text { Deficiência } \\
\text { nutricional }\end{array}$ & 59 \\
\hline Feijão de Boi & Crotalaria incana $L$ & Alguns troncos quebrados & Não identificado & 49 \\
\hline Mangiroba do Pará & & Não identificado & Não identificado & 01 \\
\hline Mangueira & Mangifera indica & Ferro, madeira & Periderme desidratada & 01 \\
\hline Mufumbo & Combretum leprosum & Cortes extravagantes & Periderme desidratada & 03 \\
\hline \multirow[t]{2}{*}{ Mororó } & Bauhini aforficata & Cortes extravagantes & Periderme desidratada & 04 \\
\hline & & & Periderme desidratada & 06 \\
\hline Mamão & Caric apapaya $L$. & Ferro, madeira & & \\
\hline Marmeleiro & Cydonia oblonga & $\begin{array}{l}\text { Presença de lixo, cortes } \\
\text { extravagantes }\end{array}$ & $\begin{array}{l}\text { Desidratação, } \\
\text { periderme }\end{array}$ & 2201 \\
\hline Nim & $\begin{array}{l}\text { Azadirachta indica A. } \\
\text { Juss }\end{array}$ & Poda inadequada & Periderme desidratada & 16 \\
\hline Orelha de Macaco & Prosopis ruscifalia & Presença de lixo & $\begin{array}{l}\text { Presença de fungos, } \\
\text { abelhas e cupins }\end{array}$ & 895 \\
\hline Pau Ferro & Caesalpinia férrea & Cortes & Periderme desidratada & 02 \\
\hline Pau D'arco roxo & $\begin{array}{l}\text { Tabebuia } \\
\text { serratifolia } \\
\text { (Vahl) Nich. }\end{array}$ & Não identificado & $\begin{array}{l}\text { Deficiência } \\
\text { nutricional }\end{array}$ & 01 \\
\hline Pau Serrote & $\begin{array}{l}\text { Hoffmanseggia } \\
\text { Falcaria }\end{array}$ & Cortes agressivos & Periderme desidratada & 72 \\
\hline
\end{tabular}




\begin{tabular}{|c|c|c|c|c|}
\hline Mufunho & Não encontrado & Não identificado & Não identificado & 01 \\
\hline Pinheiro & Araucaria angustifólia & Não identificado & Não identificado & 56 \\
\hline Pinhão Branco & Jatropha molissima & Plásticos & Periderme desidratada & 252 \\
\hline Romã & $\begin{array}{l}\text { Punica granatum. } \\
\text { Lineu }\end{array}$ & Cortes & Periderme desidratada & 02 \\
\hline Não identificada & & Cortes extravagantes & Presença cochonila & 03 \\
\hline Total Geral & & & & 5.333 \\
\hline
\end{tabular}

\section{2 - Setores de Caprinocultura}

De acordo com a (tabela 2), no setor de caprinocultura foram catalogadas um total de plantas 6.036, representando 44,13\% do total de plantas. Sendo o marmeleiro (Cydonia oblonga) a espécie de maior freqüência com (2.153 plantas), seguido a jurema (Mimosa hostilis) com 1.003 plantas , o mororó (Bauhinia ungullata) com 939 plantas, a jurema preta (Mimosa tenuiflora Cydonia oblonga) com 570 plantas, o mufumbo (Combretum leprosum) com 534 plantas.

De acordo com o levantamento qualitativo, a (tabela 2) os principais vandalismo encontrados foram : cortes agressivos, presença de lixo. Os principais problemas de fitossanidade encontrados foram: periderme desidratada e ataque de cupins.

De acordo com LOMBARDI; MORAIS (2003), OLIVEIRA (2011), as árvores são consideradas essenciais em espaços urbanos, uma vez que estas influenciam na melhoria dos fatores climáticos, como um fator determinante sobre o micro clima local.

A dominância de uma espécie varia de acordo com o local e região, onde se localiza, a interferência da população local na arborização pode ser analisada, através de algumas características, a mais importante delas é a grande presença e variedade de espécies frutíferas, mostrando que há interferência da população na arborização local (LIMA, et al 2013).

Tabela 2. Levantamento qualiquantativo e fitossanitário das espécies vegetais encontrados no setor de caprinocultura do campus IV da UEPB, Catolé do Rocha-PB, 2010/2013.

\begin{tabular}{|c|c|c|c|c|}
\hline Nome Popular & Nome Cientifico & Vandalismo & Fitossanidade e nutrição & $\begin{array}{c}\mathbf{N}^{\circ} \text { de } \\
\text { Plantas }\end{array}$ \\
\hline Angico & $\begin{array}{l}\text { Anadenanthera } \\
\text { falcata }\end{array}$ & Não identificado & $\begin{array}{l}\text { Falta de nutrientes, } \\
\text { periderme desidratada }\end{array}$ & 64 \\
\hline Aroeira & Schinus Molle & Cortes agressivo & Periderme desidratada & 65 \\
\hline Cajazeira & Spondias mombin & Cortes agressivos & Deficiência nutricional & 02 \\
\hline Catingueira & $\begin{array}{l}\text { Caesalpinia } \\
\text { pyramidalis Tui. }\end{array}$ & Não identificado & Periderme desidratada & 335 \\
\hline Cumarú & Dipteryxo dorata & Não identificado & Ataque de cupins & 02 \\
\hline Jucá & Libidibia férrea & Cortes no caule & Deficiência nutricional & 11 \\
\hline
\end{tabular}


Maeli Freitas SOUSA, Mirtes R. Fernandes DUTRA, Francineide Pereira SILVA, Thiago Pereira SOUSA, Fabiana Xavier COSTA

\begin{tabular}{|c|c|c|c|c|}
\hline Juazeiro & $\begin{array}{l}\text { Ziziphus } \\
\quad \text { joazeiro } \\
\text { Mart. }\end{array}$ & Não identificado & $\begin{array}{l}\text { Deficiência nutricional, } \\
\text { periderme desidratada }\end{array}$ & 23 \\
\hline Jurema & Mimosa hostilis & Não identificado & Ataque de cupins & 1003 \\
\hline Jurema Branca & $\begin{array}{l}\text { Piptadenia } \\
\text { stipulacea }\end{array}$ & Não identificado & Periderme desidratada & 150 \\
\hline Jurema Preta & Mimosa tenuiflora & Não identificado & Periderme desidratada & 570 \\
\hline Maniçoba & $\begin{array}{l}\text { Manihotca } \\
\text { erulescens Pohl. }\end{array}$ & Não identificado & Periderme desidratada & 29 \\
\hline Marmeleiro & Cydonia oblonga & Cortes agressivos & $\begin{array}{l}\text { Periderme } \\
\text { desidratada,fungos }\end{array}$ & 2153 \\
\hline Mororó & Bauhiniaun gullata & Cortes agressivos & $\begin{array}{l}\text { Periderme desidratada, } \\
\text { fungos }\end{array}$ & 939 \\
\hline Mufumbo & Combretum leprosum & Cortes & $\begin{array}{l}\text { Periderme desidratada, } \\
\text { cupins }\end{array}$ & 534 \\
\hline Pau Branco & Auxemma Oncocalix & Não identificado & Não apresentam doenças & 04 \\
\hline Pau Ferro & Caesalpini aférrea & Cortes agressivos & Não identificado & 02 \\
\hline Pau Serrote & $\begin{array}{l}\text { Hoffmanseggia } \\
\text { Falcaria. }\end{array}$ & Boas condições & Cupins, periderme hidratada & 09 \\
\hline Pereiro & $\begin{array}{l}\text { Aspidosperma } \\
\text { pyrifolium }\end{array}$ & Não identificado & Não apresentaram doenças & 34 \\
\hline Pinhão & $\begin{array}{l}\text { Araucária } \\
\text { angustifólia }\end{array}$ & Cortes & Periderme hidratada & 78 \\
\hline Pinhão Branco & Jatropha molissima & Não identificado & Deficiência nutricional & 06 \\
\hline Pinhão Mato & & Cortes & Falta de nutrientes & 08 \\
\hline Umburana & Amburana claudii & Não identificado & Deficiência nutricional & 07 \\
\hline Total Geral & & & & 6.036 \\
\hline
\end{tabular}

\section{3- Setores e subsetores de palmas}

De acordo com a (tabela 3) no setor do plantio da cultura da palma foram catalogadas um total de plantas 1.527, representando 11,16\% do total de plantas, Sendo a jurema (Mimosa hostilis) e espécie de maior freqüência, com 584 plantas, seguido o mufumbo (Combretum leprosu) com 277 plantas, o mororo (Bauhinia ungullata) com 199 plantas, pau louro (Bauhiniaungullata) com 103 plantas, a jurema unha de gato (Uncaria tomentosa) com 52 plantas.

No levantamento qualitativo (tabela 3), os principais vandalismos verificados na pesquisa foram: cortes extravagantes e presença de lixo. Os problemas de fitossanidade encontrados foram: periderme desidratada e deficiência nutricional.

Araújo et al (2011), afirmam que diversos autores têm citado Euphorbiaceae e Cactaceae como famílias de grande representatividade em vários levantamentos florísticos realizados em áreas de Caatinga.

Segundo Maia (2012), a jurema-preta (Mimosa tenuiflora (Willd. Poir.) tem um grande potencial como planta regeneradora de solos erodidos. É uma espécie indicadora de 
uma sucessão secundária progressiva ou de recuperação e sua tendência ao longo do processo é de redução da densidade.

Tabela 3-Levantamento qualiquantativo das espécies vegetais, encontrados no setor e subsetor de palmas (área do canhão I e II e milharal) do campus IV UEPB, Catolé do RochaPB-2010/2013.

\begin{tabular}{|c|c|c|c|c|}
\hline Nome Popular & Nome Cientifico & Vandalismo & Fitossanidade e nutrição & $\begin{array}{c}\mathbf{N}^{\mathbf{o}} \text { de } \\
\text { Plantas }\end{array}$ \\
\hline Algaraba & Prosopis juliflora & Lixo,cortes & Periderme desidratada & 18 \\
\hline Angico & $\begin{array}{l}\text { Anadenanthera } \\
\text { Colubrina. }\end{array}$ & Não identificado & Deficiência nutricional & 22 \\
\hline Bugi & Alouatta Guariba. & Não identificado & Não identificado & 07 \\
\hline Carnaúba & $\begin{array}{l}\text { Coperniciaprunifer } \\
a(\text { Mill.) ... }\end{array}$ & Lixo & Periderme desidratada & 02 \\
\hline Camará & Lantana camara & Lixo & Deficiência nutricional & 01 \\
\hline Cajueiro & $\begin{array}{l}\text { Anacardium } \\
\text { occidentale }\end{array}$ & Lixo & Deficiência nutricional & 08 \\
\hline Castanhola do mato & Terminalia catappa & Não identificado & Periderme desidratada & 01 \\
\hline Cajazeira & Spondias lútea & $\begin{array}{l}\text { Cortes } \\
\text { extravagantes }\end{array}$ & Periderme desidratada & 01 \\
\hline Catingueira & $\begin{array}{l}\text { Caesalpinia } \\
\text { pyramidalisTui }\end{array}$ & Não identificado & Não identificado & 07 \\
\hline Café do mato & Cordiaecal yculata & Não identificado & Não identificada & 11 \\
\hline Feijão boi & Clotilata incana l & Não identificado & Periderme desidratada & 20 \\
\hline Goiabeira & Psidium guajava & Não identificado & Não identificado & 03 \\
\hline Goiabinha & Não encontrado & Não identificado & Não identificado & 02 \\
\hline Jaramataia & Vitexgardneriana & Não identificado & Não identificado & 05 \\
\hline Jenipapo & Genipa americana & Não identificado & Periderme desidratada & 01 \\
\hline Jurema unha de gato & Uncaria tomentosa & Não identificado & Não identificado & 52 \\
\hline Juazeiro & Ziziphusjoazeiro Mart & Lixo & Periderme desidatada & 24 \\
\hline Jucá & Caesalpinia Ferrea & Não identificado & Não identificado & 03 \\
\hline Jurema & Mimosa hostilis & Cortes & Periderme desidratada & 584 \\
\hline Jurema preta & $\begin{array}{l}\text { Mimosa tenuiflora } \\
\text { (Mart.) Benth }\end{array}$ & aNão identificado & Fungos cochinila & 05 \\
\hline Jurubeba & $\begin{array}{l}\text { Solanumpani culatum } \\
\text { L. }\end{array}$ & Não identificado & Periderme desidratada & 04 \\
\hline Leucena & $\begin{array}{l}\text { Leucaena leucocep } \\
\text { hala. }\end{array}$ & Lixo & Deficiência nutricional & 09 \\
\hline Linho & $\begin{array}{l}\text { Linum } \\
\text { Usitatissimum }\end{array}$ & Cortes agressivos & Deficiência nutricional & 18 \\
\hline Maniçoba & $\begin{array}{l}\text { Manihotcae } \\
\text { rulescensPohl. }\end{array}$ & Não identificado & Não identificado & 16 \\
\hline Marmeleiro & Cydonia oblonga & $\begin{array}{l}\text { Cortes } \\
\text { extravagantes }\end{array}$ & Periderme desidratada & 199 \\
\hline $\begin{array}{l}\text { Manjiroba brava do } \\
\text { mato }\end{array}$ & $\begin{array}{l}\text { Origanum majorana } \\
L\end{array}$ & Não identificado & Não identificado & 01 \\
\hline Mororó & $\begin{array}{l}\text { Bauhiniaun gullata } \\
\text { Link }\end{array}$ & Não identificado & Não identificado & 21 \\
\hline Mufumbo & Combretum leprosum & Cortes extravagantes & Periderme desidratada & 277 \\
\hline $\mathrm{Nim}$ & Azadirachta & Não identificado & Não identificado & 01 \\
\hline
\end{tabular}


Maeli Freitas SOUSA, Mirtes R. Fernandes DUTRA, Francineide Pereira SILVA, Thiago Pereira SOUSA, Fabiana Xavier COSTA

\begin{tabular}{|c|c|c|c|c|}
\hline Otiti do mato & Licania tomentosa & \multicolumn{2}{|c|}{ Cortes extravagantes Periderme desidratada } & 03 \\
\hline Oticica & Licania Rígida & Lixo & Periderme desidratada & 04 \\
\hline Pau D’arco & $\begin{array}{l}\text { Tabebuia } \\
\text { serratifolia } \\
\qquad(\text { Vahl) Nich }\end{array}$ & Lixo & Fungos e cupins & 04 \\
\hline Pau serrote & $\begin{array}{l}\text { Hoffmanseggia } \\
\text { Falcaria. }\end{array}$ & Lixo & Periderme desidratada & 15 \\
\hline Pau são João & Não encontrada & Não identificado & Não identificado & 03 \\
\hline Pau branco & Auxemma Oncocalix & Não identificado & Periderme desidratada & 10 \\
\hline Pau louro & Bauhiniaun gullata & Não identificado & Não identificado & 103 \\
\hline Pinhão do mato & $\begin{array}{l}\text { Araucária } \\
\text { angustifolia (Bertol.) }\end{array}$ & Lixo & Periderme desidratada & 18 \\
\hline Quebra faca & $\begin{array}{l}\text { Cochlosperm } \\
\text { umregium }\end{array}$ & Não identificado & Periderme desidratada & 01 \\
\hline Sabonete & Sapinduss aponaria & Não identificado & Periderme desidratada & 05 \\
\hline Trapiá & Crateva Tapia & Não identificado & Periderme desidratada & 04 \\
\hline Velame & $\begin{array}{l}\text { Croton } \\
\text { heliotropiifolius }\end{array}$ & Não identificado & Periderme desidratada & 04 \\
\hline Total Geral & & & & 1.527 \\
\hline
\end{tabular}

\section{4- Setor de olericultura}

No setor de olericultura foram catalogados um total de 88 plantas, representando 0,64\% do total encontrado. (De acordo com a (tabela 4) a leucena (Leucaena leucocephala) foi a espécie de maior frequiência com 35 plantas), em seguido o feijão de boi (Crotalaria incana) com 10 plantas, a algaroba (Prosopis Juliflora) com 8 plantas.

No levantamento qualitativo (tabela 4) o principal vandalismo encontrado foi: cortes extravagantes. O principal problema de fitossanidade encontrado foi: periderme desidratada.

Para Chaves et. al (2013), a presença de espécies invasoras traz modificações nos ciclos biológicos, competição com as espécies nativas, redução da biodiversidade, mudanças nas freqüências e intensidade de incêndios, e aumento de pragas na região.

Padrão semelhante foi registrado por Sousa et al (2010), que observaram ainda que as condições abióticas distintas ao longo de um gradiente semelhante, com variações de relevo e solo, a partir do curso d'água até o tabuleiro, influenciaram na composição das espécies vegetais predominantes numa área.

TABELA 4- Levantamento qualiquantativo e fitossanitário das espécies vegetais encontrados no setor de Olericultura do campus IV da UEPB, Catolé do Rocha-PB, 2010/2013. 


\begin{tabular}{|c|c|c|c|c|}
\hline $\begin{array}{l}\text { Nome } \\
\text { Popular }\end{array}$ & $\begin{array}{c}\text { Nome } \\
\text { Cientifico }\end{array}$ & Vandalismo & $\begin{array}{c}\text { Fitossanidade e } \\
\text { nutrição }\end{array}$ & $\begin{array}{c}\mathrm{N}^{\mathbf{o}} \text { de } \\
\text { Plantas }\end{array}$ \\
\hline Algaroba & Prosopi sJuliflora. & Cortes extravagantes & Periderme desidratada & 08 \\
\hline Barandão & Não encontrado & Não identificado & Não encontrado & 05 \\
\hline Cajueiro & Anacardiumo ccidentale & Cortes extravagantes & Periderme desidratada & 01 \\
\hline Carnaúba & Copernicia prunifera) & Cortes & Periderme desidratada & 01 \\
\hline Catingueira & Caesalpini apyramidalisTui. & Lixos & Periderme desidratada & 01 \\
\hline Coqueiro & Cocos nucifera & Lixos & Periderme desidratada & 06 \\
\hline Feijão boi & Crotalaria incana $l$ & Cortes & $\begin{array}{l}\text { Deficiência } \\
\text { nutricional }\end{array}$ & 10 \\
\hline Goiabera & Psidium guajava & Lixo & Ataque de formigas & 01 \\
\hline Jaramatia & Vitex gardneriana & Não identificado & Não encontrado & 01 \\
\hline Jenipapo & Genipa americana & Não identificado & Periderme desidratada & 01 \\
\hline Jurema & Mimosa hostilis & Não identificado & Periderme desidratada & 02 \\
\hline Leucena & Leucaena leucocephala. & Cortes extravagantes & Periderme desidratada & 35 \\
\hline Limoeiro & Citrus limon & Lixo & Fungos cochonila & 01 \\
\hline Mangueira & Mangifera indica & Cortes profundos & Periderme desidratada & 01 \\
\hline Marmeleiro & Cydonia oblonga & Não identificado & Não identificado & 01 \\
\hline Mororó & Bauhiniaun gullata Link & Cortes extravagantes & Periderme desidratada & 02 \\
\hline Mufumbo & Combretum leprosum & Cortes & Periderme desidratada & 04 \\
\hline Otiticia & Licania rígida & Não identificado & Não identificado & 03 \\
\hline Pau d'arco & $\begin{array}{l}\text { Tabebuia serratifolia (Vahl) } \\
\text { Nich }\end{array}$ & Não identificaddo & Não identificado & 01 \\
\hline Pau do Pará & $\begin{array}{l}\text { Tabebuia serratifolia (Vahl) } \\
\text { Nich }\end{array}$ & Cortes & Periderme desidratada & 01 \\
\hline Pinheira & Araucaria angustifólia & Lixo & Periderme ressecada & 01 \\
\hline Sabonete & Sapindus saponaria & Não encontrado & Periderme desidratada & 01 \\
\hline TOTAL & & & & 88 \\
\hline
\end{tabular}

\subsection{Margens do riacho Agom}

Nas margens do riacho Agom foi catalogado um total de 586 plantas, representando 4,28\% do total encontrados. De acordo com a (tabela 5) jurema unha de gato (Mimosa hostilis Benth) foi a espécie de maior frequiência, com 96 plantas, seguido o café do mato (Cordiae calyculata) com 91 plantas, a fava de boi (Crotalaria incana) com 84 plantas, e a jurema preta (Mimosa hostilis Benth) com 41 plantas.

No levantamento qualitativo (tabela1), o principal vandalismo encontrado foi: cortes. Já os problemas de fitossanidade foram: periderme desidratada e deficiência nutricional.

Entretanto, para que se tenha um ambiente ecologicamente equilibrado é necessária à realização de um planejamento prévio para a escolha de espécies adequadas para a arborização, dando-se prioridade a espécies nativas que sejam adaptadas ao clima da região e que desempenhem uma função ecológica importante para o bioma (BRUN et al., 2007 ;LOBODA; DE ANGELES, 2009). 
De acordo com Araújo et. al (2012), a maior diversidade de espécies de árvores na paisagem de um determinado local se faz necessária para garantir o máximo de proteção contra pragas e doenças, sendo que,avaliando qualitativamente a composição vegetal do Parque Internacional em Sant'ana do Livramento verificaram que $34,82 \%$ da composição arbórea do Parque estavam infestados por cupins, sendo a farinheira (Albizia hasslerii), pitombeira (Talisia esculenta) e jatobá (Hymenaea stigonocarpa) as espécies com maiores incidências. Havendo também a presença de hemiparasitas (erva de - passarinho) em seus ramos.

TABELA 5- Levantamento quantiqualitativo e fitossanitário das espécies vegetais encontrados no setor do Riacho Agom do campus IV da UEPB, Catolé do Rocha-PB, 2010/2013.

\begin{tabular}{|c|c|c|c|c|}
\hline $\begin{array}{c}\text { Nome } \\
\text { Popular }\end{array}$ & $\begin{array}{c}\text { Nome } \\
\text { Cientifico }\end{array}$ & Vandalismo & $\begin{array}{c}\text { Fitossanidade e } \\
\text { nutrição }\end{array}$ & $\begin{array}{c}\mathbf{N}^{\circ} \text { de } \\
\text { Plantas }\end{array}$ \\
\hline Algaroba & Prosopis Juliflora & Não identificado & Não identificado & 15 \\
\hline Barandão & Não encontrado & L ixo & Fungos & 37 \\
\hline Café do mato & Cordiae calyculata & Não identificado & Fungos & 91 \\
\hline Cajarana & Spondias Lutea & Cortes agressivos & $\begin{array}{l}\text { Periderme } \\
\text { desidratada }\end{array}$ & 14 \\
\hline Cajazeira & Spondia smombin & Não identificado & Cupins & 02 \\
\hline Cajueiro & Anacardiumo ccidentale & Cortes agressivos & Cupins & 05 \\
\hline Carnaúba & Copernici aprunifera & Não identificado & Não identificado & 03 \\
\hline Fava de boi & Crotalaria incana $l$ & Não identificado & Ataque de fungos & 84 \\
\hline Ingazeira & Íngaedulis Mart & Não identificado & Fungos & 01 \\
\hline Jaramataia & $\begin{array}{l}\text { Vitex } \\
\text { Gardneriana }\end{array}$ & Não identificado & Não identificado & 07 \\
\hline Juazeiro & Ziziphusjo azeiro Mart & Não identificado & $\begin{array}{l}\text { Periderme } \\
\text { desidratada }\end{array}$ & 11 \\
\hline Jucá & Caesalpinea Ferra & Não identificado & Fungos & 01 \\
\hline Jurema & $\begin{array}{l}\text { Mimosa tenuiflora (Will.) } \\
\text { Poire }\end{array}$ & Cotes & $\begin{array}{l}\text { Periderme } \\
\text { desidratada }\end{array}$ & 05 \\
\hline $\begin{array}{l}\text { Jurema unha de } \\
\text { gato }\end{array}$ & Mimosa hostilis Benth & Cortes & $\begin{array}{l}\text { Periderme } \\
\text { desidratada }\end{array}$ & 96 \\
\hline Jurema preta & Mimosa hostilis Benth. & Não identificado & Não identificado & 41 \\
\hline Linho & Linumu sitatissimum L. & Cortes extravagantes & $\begin{array}{l}\text { Periderme } \\
\text { desidratada }\end{array}$ & 20 \\
\hline Maniçoba & Maniho tesculenta & Não identificado & Não identificado & 13 \\
\hline $\begin{array}{l}\text { Manjiroba do } \\
\text { Pará }\end{array}$ & Origanum majorana $L$ & Tronco quebrado & $\begin{array}{l}\text { Deficiência } \\
\text { nutricional }\end{array}$ & 36 \\
\hline Marinzeiro & Não encontrado & Não identificado & Não identificado & 04 \\
\hline Marmeleiro & Cydonia oblonga & Não identificado & Não identificado & 03 \\
\hline Mororó & Bauhiniaun gullata Link. & Não identificado & $\begin{array}{l}\text { Periderme } \\
\text { desidratada }\end{array}$ & 03 \\
\hline Mufumbo & Combretum leprosum) & Não identificado & $\begin{array}{l}\text { Periderme } \\
\text { desidratada }\end{array}$ & 09 \\
\hline
\end{tabular}


Maeli Freitas SOUSA, Mirtes R. Fernandes DUTRA, Francineide Pereira SILVA, Thiago Pereira SOUSA, Fabiana Xavier COSTA

\begin{tabular}{|c|c|c|c|c|}
\hline Mulumgo & Erythrina mulungu & Não identificado & Fungos & 05 \\
\hline $\mathrm{Nim}$ & Azadirachta indica A. Juss & Não identificado & Não identificado & 01 \\
\hline Oticica & Licaniaaarigida Benth & Não identificado & Fungos & 14 \\
\hline $\begin{array}{l}\text { Orelha de } \\
\text { macaco }\end{array}$ & Enterolobium contortisiliquum & Lixo & Cupim & 02 \\
\hline Pau d'arco & $\begin{array}{l}\text { Tabebuia serratifolia (Vahl) } \\
\text { Nich }\end{array}$ & Não identificado & Cupim & 01 \\
\hline Pau do Pará & Tabebuia serratifolia & Não identificado & Não identificado & 03 \\
\hline Pau ferro & Caesalpinia férrea & Não identificado & Cupim & 03 \\
\hline Pinhão branco & Jatropha molissima & Cortes agressivos & $\begin{array}{l}\text { Periderme } \\
\text { desidratada }\end{array}$ & 02 \\
\hline Pinhão roxo & Jatrophacurcas $L$ & Não identificado & Não identificado & 01 \\
\hline Sabiá & $\begin{array}{l}\text { Mimosa caesalpiniaefolia } \\
\text { Benth }\end{array}$ & Não identificado & Não identificado & 12 \\
\hline Trapiá & Crateva Tapia. & Cortes & $\begin{array}{l}\text { Periderme } \\
\text { desidratada }\end{array}$ & 23 \\
\hline TOTAL & & & & 586 \\
\hline
\end{tabular}

\section{CONCLUSÕES}

-Há alta diversidade de espécies nas áreas arborizadas do Campus IV da UEPB;

-A alta diversidade de espécies, apesar de grandes problemas de vandalismo e fitossanidade encontrados nos setores avaliados, indica qualidade na estrutura física e ambiental;

-O conhecimento da diversidade florística é de fundamental importância para a estrutura vegetativa;

-O marmeleiro foi à espécie mais encontrada no Campus IV.

\section{REFERÊNCIAS}

ARAUJO, A. C. B.; GRACIOLI, C. R.; GRIMM, E. L.; LONGH, S. J. Avaliação da florística, do porte e da fitossanidade atual da arborização do parque internacional em Santana do Livramento/Rivera, Brasil/Uruguai. Revista da Sociedade Brasileira de Arborização Urbana - REVSBAU, Piracicaba-SP, v.7, n.1, p. 112-125, 2011.

ARAUJO, K. D.; PARENTE, H. N.; SILVA, É. É.; RAMALHO, C. I.; DANTAS, R. T.; ANDRADE, A. P.; SILVA, D. S. Levantamento florístico do estrato arbustivo-arboreo em áreas contíguas de caatinga no cariri paraibano. Revista Caatinga, vol. 23, núm. 1, eneromarzo, 2010, pp. 63-70, Universidade Federal Rural do Semi-Árido, Brasil.

ARAUJO, Avaliação da florística, do porte e da fitossanidade atual da arborização do parque internacional em Santana do Livramento/Rivera, Brasil/Uruguai. Revista da Sociedade Brasileira de Arborização Urbana - REVSBAU, Piracicaba-SP, v.7, n.1, p. 112-125, 2012. 
BRUN, E. J. B.; BRUN, F. G. K. Arborização urbana \& qualidade de vida. Revista CREARS, Porto Alegre, n. 18, p. 27, fev. 2007.

CENCI, B. T.; DORNELES, L. T.; SIMONI, E. L.; FRIZON, S. M.; TRAVI. V. H. Composição da flora arbórea e arborescente no jardim botânico de Bento Gonçalves, Rio Grande do Sul, Brasil. Revista Árvore, Viçosa-MG, v.37, n.1, p.137-149, 2013.

CHAVES, A. D. C. G.; SANTOS, R. M. S.; SANTOS, J. O.; FERNANDES, A. A.; MARACAJÁ, P. B. A importância dos levantamentos florístico e fitossociológico para a conservação e preservação das florestas. Revista ACSA - OJS, V. 9, n. 2, p. 42-48, abr - jun, 2013.

DIAS, Genebaldo Freire. Educação Ambiental: Princípios e Práticas. $3^{\text {a }}$ ed. São Paulo; $3^{\text {a }}$ ed. São Paulo :Gaia , 2004.

GURGEL, J. M.; ROCHA, A. M. B. D.; AQUINO, J. T. D. Inventário florístico do município de Cajazeiras PB área urbana realizado pela SUMMAC Superintendência municipal de meio ambiente de Cajazeiras. In: I Simpódio paraibano de Meio Ambiente; III Encontro de Biologia. 1., 2009. Cajazeiras, Paraíba. Anais...., Paraíba, Brasil, 2009.

LIMA, P. C. C.; CARVALHO, J. A.; AVILA, R. G.; SILVA, D. V.; SOBREIRA. B. G. Avaliação qualiquantitativa da arborização urbana da avenida Dr. Luiz Intro caso Filho em Carmo do Rio Claro-MG. in: X Congresso Nacional de Meio Ambiente de Poços de Caldas. V.5, N.1. Anais...Poços de Caldas-MG, 2013.

LOBODA, C. R.; DE ANGELIS, B. L. D. Áreas verdes públicas urbanas: conceitos, usos e funções. Ambiência, v. 1, n. 1, p. 125-139, 2009.

LOMBARDI, J. A.; MORAIS, P. O. Levantamento florístico das plantas empregadas na arborização do campus da Universidade Federal de Minas Gerais, Belo

Horizonte-MG. Lundiana-International Journal of Biodiversity, v. 4, n. 2, p. 83-88, 2003.

\section{LÜTZ, MONIQUE DAMO. PSICOLOGIA AMBIENTAL E EDUCAÇÃO}

AMBIENTAL: contribuições para a transformação sócio-ambiental. Disponível em: http://siaibib01.univali.br/pdf/Monique\%20Damo\%20Lutz.pdf.Acesso em: 3 de janeiro de 2014.

MAIA, GerdaNickel. Caatinga: árvores e arbustos e suas utilidades. São Paulo: D\&Z Computação Gráfica e Editora, 2012.

OLIVEIRA, A. S. D. E. Influência da vegetação arbórea no microclima e uso de praças públicas. 162 f. Tese (Doutorado em Física Ambiental) - Instituto de Física,Universidade Federal do Mato Grosso, Cuiabá, 2011. 
Planeta sustentável. Disponível em: http://planetasustentavel.abril.com.br/.Acesso em: 17 de dezembro de 2013.

Política Nacional do Meio Ambiente. Disponível em: www.paisagismodigital.com.brAcesso em 01de janeiro de 2013.

SOUZA, J. A. N.; RODAL, M. J. N. Levantamento floristico em um trecho de vegetação ripária de caatinga no Rio Pajeú, Floresta/Pernanbuco. Revista Caatinga, vol. 23, núm. 4, outubro-dezembro, 2010, pp. 54-62, Universidade Federal Rural do Semi-Árido, Brasil. 